\title{
Optical Engineering
}

\section{New elliptical parallax barrier pattern to reduce the cross talk caused by light leakage}

\author{
Yongsik Jung \\ Jong-Man Kim \\ Jongbin Kim \\ Hyunsik Sung \\ Sung-Wook Min \\ Seung-Woo Lee
}

\section{SPIE.}




\title{
New elliptical parallax barrier pattern to reduce the cross talk caused by light leakage
}

\author{
Yongsik Jung, Jong-Man Kim, Jongbin Kim, Hyunsik Sung, Sung-Wook Min, ${ }^{\star}$ and Seung-Woo Lee* \\ Kyung Hee University, Department of Information Display and Advanced Display Research Center, 1 Hoegi-dong, Dongdaemoon-gu, \\ Seoul 130-701, Republic of Korea
}

\begin{abstract}
This paper proposes a parallax barrier with an elliptical pattern that reduces the cross talk caused by light leakage from adjacent subpixels in autostereoscopic three-dimensional (3-D) displays. To find the optimum size of the elliptical barrier pattern, the relationship between the reduction of the light leakage and that of the luminance is analyzed. In addition, we analyze the relationship between the cross talk and the luminance. By using these relationships, we propose an optimum size of the ellipse. An autostereoscopic 3-D display with the elliptical barrier is compared with 3-D displays with the slanted barrier and the rectangular one. The measured cross talk of the slanted-type 3-D display whose pixel size is $98 \times 294 \mu \mathrm{m}$ was $57 \%$. However, the cross talk of the ellipse-type 3-D display was 32\% at the similar luminance condition when the minor and major axes are 92 and $278 \mu \mathrm{m}$, respectively. For generalization, we investigate autostereoscopic 3-D displays with different pixel sizes and different viewing distances. We find the optimum area of the ellipse is $70 \%$ of the subpixel area to reduce the cross talk. $\odot$ The Authors. Published by SPIE under a Creative Commons Attribution 3.0 Unported License. Distribution or reproduction of this work in whole or in part requires full attribution of the original publication, including its DOI. [DOI: 10.1117/1.OE.53.2.025101]
\end{abstract}

Keywords: autostereoscopic display; cross talk; light leakage.

Paper 131571 received Oct. 14, 2013; revised manuscript received Jan. 21, 2014; accepted for publication Jan. 23, 2014; published online Feb. 14, 2014.

\section{Introduction}

These days, many types of autostereoscopic display devices have been developed. One optical technology used in autostereoscopic displays is a parallax barrier. Autostereoscopic displays with the parallax barrier have superior three-dimensional (3-D) display characteristics and low costs. ${ }^{1,2}$ Autostereoscopic displays provide views for multiple viewers to perceive stereoscopic images without glasses. ${ }^{3,4} \mathrm{~A}$ barrier with a slanted pattern has been generally used for autostereoscopic displays because it features a balanced resolution in both the horizontal and vertical directions and reduced moire artifacts on the display screen. However, a conventional autostereoscopic display with the slanted parallax barrier suffers from undesired cross talk that deteriorates the stereoscopic image quality. ${ }^{5-8}$ There are many kinds of factors that influence the cross talk in autostereoscopic displays. Kooi ${ }^{9}$ found that the contrast of the display and binocular disparity of the 3-D images are the important factors that determine the cross talk. He found that cross talk is less visible when the displays and 3 -D images have a high contrast ratio $(100: 1)$ and a reasonable binocular disparity (40 arc min), respectively. Kooi and Toet ${ }^{10}$ found that the vertical disparity of human eyes and blur of the 3-D images affect the visual comport. The cross talk becomes more visible with increasing vertical disparity and sharpness of the 3-D images. However, the most important factor that influences cross talk in autostereoscopic displays with parallax barrier is the light from adjacent subpixels. ${ }^{11,12}$ Generally, pixels of the display are rectangular, thus a slanted barrier cannot completely block the light from adjacent subpixels as shown in Fig. 1(a). In order to solve this problem, many methods have been proposed

*Address all correspondence to: Sung-Wook Min, E-mail: mins@khu.ac.kr; Seung-Woo Lee, E-mail: seungwoolee@khu.ac.kr such as modified pixel layout, ${ }^{6}$ fusion of viewing zones, ${ }^{13}$ and so on. As shown in Fig. 1(b), Mashitan et al. proposed a multiview autostereoscopic display with a rectangular barrier pattern. ${ }^{14}$ However, they did not study the cross talk characteristics of the rectangular barrier pattern. We expect that the rectangular barrier pattern can solve the structural limitation of the conventional slanted barrier pattern. In our experiment, we verified that the rectangular barrier pattern is superior to the conventional slanted barrier pattern with regard to cross talk. However, the autostereoscopic displays with the rectangular barrier pattern also suffer from cross talk caused by unwanted light leakage from adjacent subpixels as shown in Fig. 2. The unwanted light leakage mainly falls into three classifications: first is light leakage from vertically and horizontally adjacent pixels (e.g., to the second pixels from the first and third), second is from diagonally adjacent pixels (e.g., to second pixels from the fourth and sixth), and the third is the light leakage from adjacent pixels of the same viewpoint (e.g., to second from the other second pixels). The cross talk caused by light leakage from adjacent subpixels must be resolved because cross talk is the most critical issue impeding the development of 3-D displays.

In this paper, we analyze how the cross talk is influenced by the various barrier patterns and various sizes of barrier patterns. Finally, we propose a new parallax barrier pattern and its optimum size that would resolve the cross talk caused by light leakage.

\section{Device Structure}

We used a 19 -in. SXGA $(1280 \times 1024)$ patterned-vertical alignment (PVA) liquid crystal display (LCD) for our experiments. The size of a subpixel is approximately $98 \times 294 \mu \mathrm{m}$. The horizontal and vertical lengths of black matrix are 11 


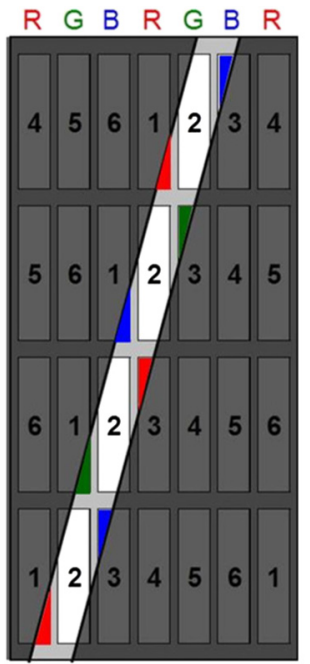

(a)

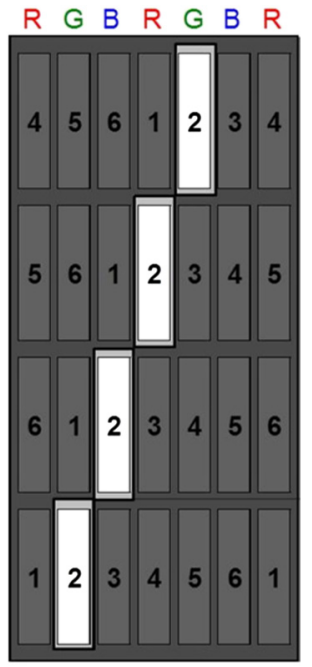

(b)
Fig. 1 Autostereoscopic displays comprising the patterned-vertical alignment panel and a parallax barrier. The patterns of the barriers of (a) are slanted and (b) are rectangular.

and $21 \mu \mathrm{m}$ as shown in Fig. 3(a). The optical structure of the autostereoscopic 3-D display is shown in Fig. 3(b). The LCD panel is located under the parallax barrier with a gap, $g$, of $0.75 \mathrm{~mm}$. The distance between the right and left sweet spots, $b$, must be equal to the interpupillary distance, once again, assumed to be $65 \mathrm{~mm} .{ }^{15}$ Thus, the optimum viewing distance, $d$, is determined as $50 \mathrm{~cm}^{15}$

\section{New Parallax Barrier Pattern with Elliptical Shape}

In the case of the slanted barrier pattern, light leakage is not the main reason for cross talk because of the structural limitation as shown in Fig. 1(a). The main reason for cross talk in the slanted barrier pattern is that the slanted barrier cannot completely cover the subpixel's boundaries. In the rectangular barrier pattern, the main reason for the cross talk is the light leakage from adjacent subpixels. To solve this problem of light leakage in the rectangular barrier pattern, we need to

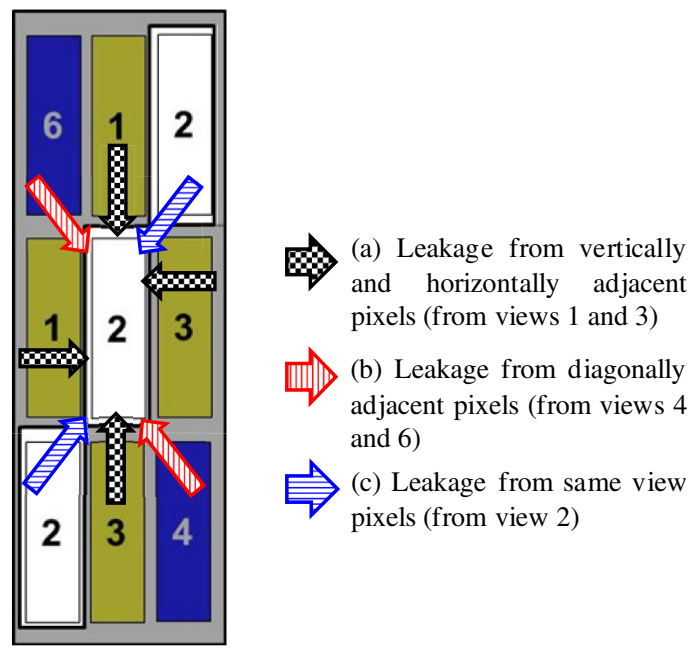

Fig. 2 Unwanted light leakage from adjacent subpixels. Light leakage from (a) vertically and horizontally adjacent pixels, (b) diagonally adjacent pixels, and (c) the same view pixels.

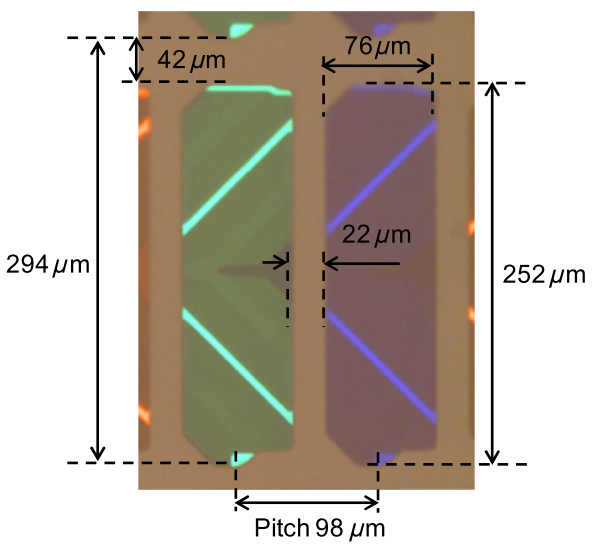

(a)

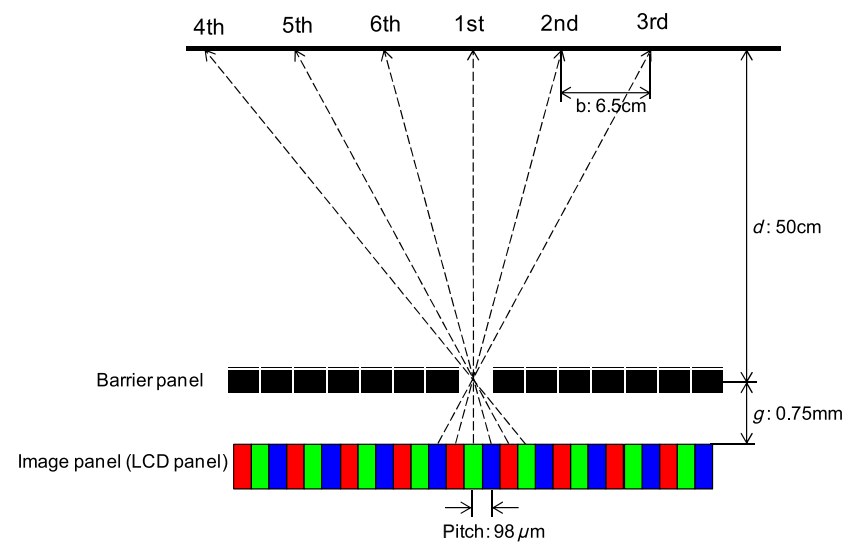

(b)

Fig. 3 Schematic diagram of an autostereoscopic display (a) Dimensions of an LCD subpixel. (b) Optical structure of the autostereoscopic three-dimensional display.

carefully investigate it. Figure 4 shows microscopic images of how the light leakage depends on the test image pattern. In Fig. 4, only the area of the fourth view is open and the other area for the remaining views is blocked. The size of the open area is about $98 \times 294 \mu \mathrm{m}$ which is the same size as a single subpixel as shown in Fig. 3(a). Thus, it is natural to expect images through the fourth view only at the fourth view point. At this time, the distance between parallax barrier and LCD panel is $0.75 \mathrm{~mm}(\mathrm{~g})$ which is the same as condition to Fig. 3(b). We took a microscopic image at the position normal to $4 \mathrm{C}$ pixel on the barrier. The subpixel $4 \mathrm{C}$ is turned off in Fig. 4. Figure 4(a) shows a microscopic image of when two red subpixels that are located at the upper and lower positions in relation to the subpixel $4 \mathrm{C}$ are turned on. If the light leakage from vertically adjacent pixels does not exist, 4C pixel has to display a pure black image as shown in ideal image of Fig. 4(a). However, we can easily observe the light leakage from vertically adjacent pixels as shown in actual photo of Fig. 4(a). In Fig. 4(b), we can also observe the light leakage of when a subpixel at each of the left and right side of the subpixel 4C are turned on. Also, we cannot neglect the light leakage from diagonally adjacent pixels as shown in Figs. 4(c) and 4(d). As a result, we can find that we need to consider two things to reduce the light leakage from adjacent subpixels: The first is the horizontal and vertical lengths of the barrier pattern. The second is the shape of the barrier. The light leakage from 


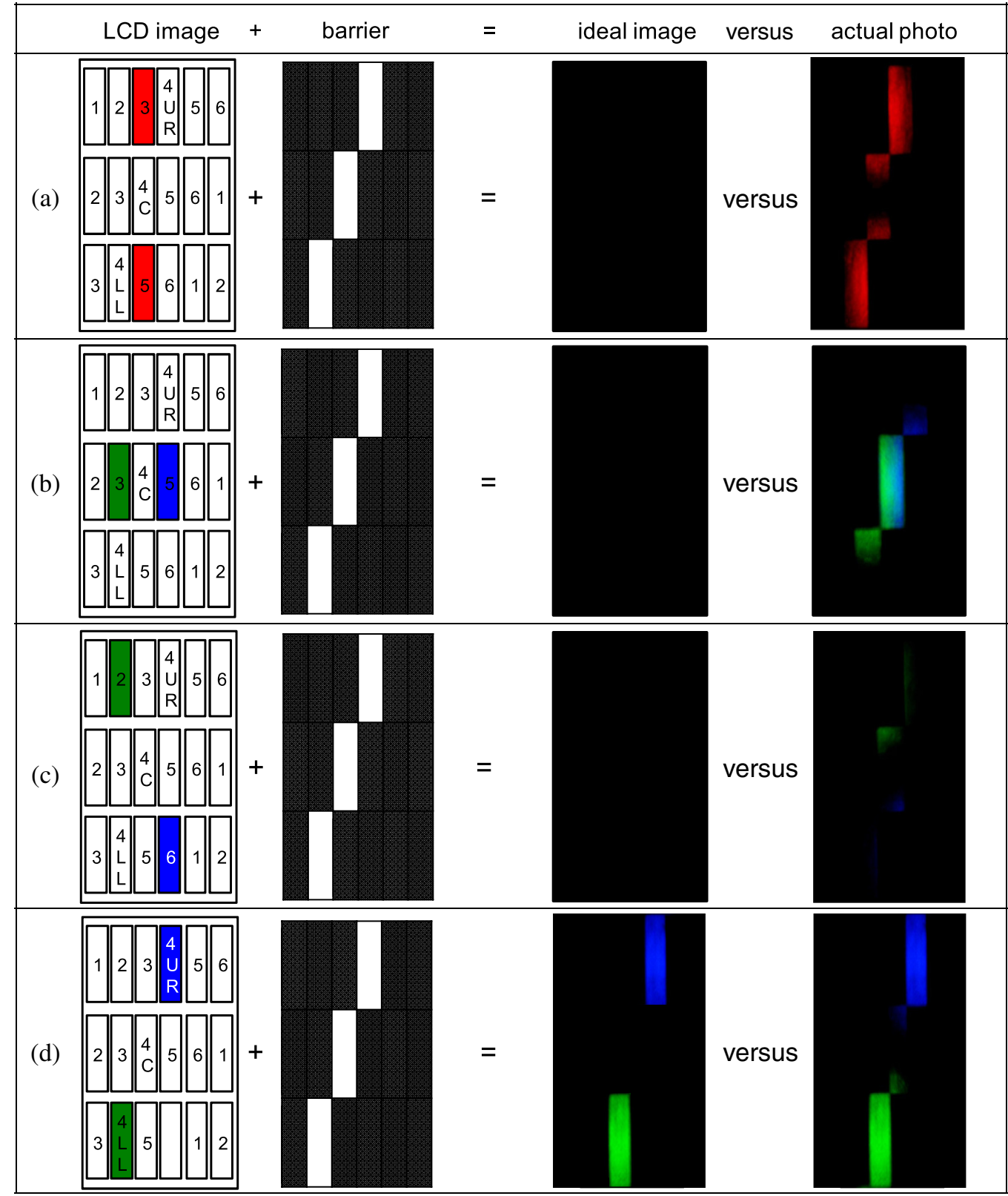

Fig. 4 Microscopic images on an autostereoscopic display depending on LCD screen images when only the fourth view is open and the pixel $4 \mathrm{C}$ is black. (a) Vertically adjacent pixels affecting the pixel 4C. (b) Horizontally adjacent green and blue pixels affecting the pixel 4C. (c) Diagonally adjacent green and blue pixels affecting the pixel 4C. (d) Green (4UR) and blue (4LL) pixels in the fourth view affecting the pixel 4C.

\begin{tabular}{|c|c|c|c|c|c|c|}
\hline $\begin{array}{c}\text { Slanted pattern } \\
\text { No. }\end{array}$ & 1 & 2 & 3 & 4 & 5 & 6 \\
\hline $\begin{array}{c}\text { Microscopic } \\
\text { image }\end{array}$ & & & & & & \\
\hline Width $(\mu \mathrm{m})$ & 108 & 98 & 88 & 75 & 64 & 53 \\
\hline Cross talk (\%) & 57.03 & 52.88 & 50.8 & 47.22 & 45.1 & 44.63 \\
\hline $\begin{array}{c}\text { Luminance } \\
\left(\mathrm{cd} / \mathrm{m}^{2}\right)\end{array}$ & 14.50 & 13.20 & 12.42 & 10.80 & 9.60 & 8.00 \\
\hline
\end{tabular}

Fig. 5 Microscopic images and measured cross talk of the slanted barrier depending on the various pattern sizes. 


\begin{tabular}{|c|c|c|c|c|c|c|}
\hline $\begin{array}{c}\text { Rectangle } \\
\text { pattern No. }\end{array}$ & 1 & 2 & 3 & 4 & 5 & 6 \\
\hline $\begin{array}{c}\text { Microscopic } \\
\text { image }\end{array}$ & & & & & & \\
\hline Size $(\mu \mathrm{m})$ & $98 \times 294$ & $86 \times 262$ & $76 \times 252$ & $66 \times 242$ & $56 \times 232$ & $46 \times 222$ \\
\hline Cross talk (\%) & 44.37 & 35.72 & 34.17 & 32.94 & 32.16 & 31.11 \\
\hline $\begin{array}{c}\text { Luminance } \\
\left(\mathrm{cd} / \mathrm{m}^{2}\right)\end{array}$ & 15.29 & 13.27 & 11.94 & 10.14 & 8.52 & 7.04 \\
\hline
\end{tabular}

Fig. 6 Microscopic images and measured cross talk of the rectangular barrier depending on the various pattern sizes.

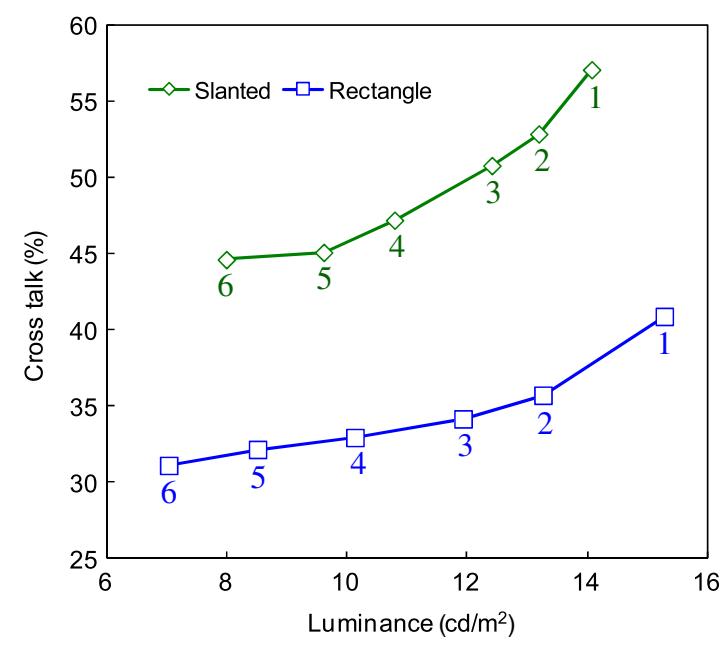

Fig. 7 Summarized results of cross talk depending on the various sizes of the slanted and rectangular barrier patterns at the same luminance (the numbers represent the pattern numbers in Figs. 5 and 6 ).

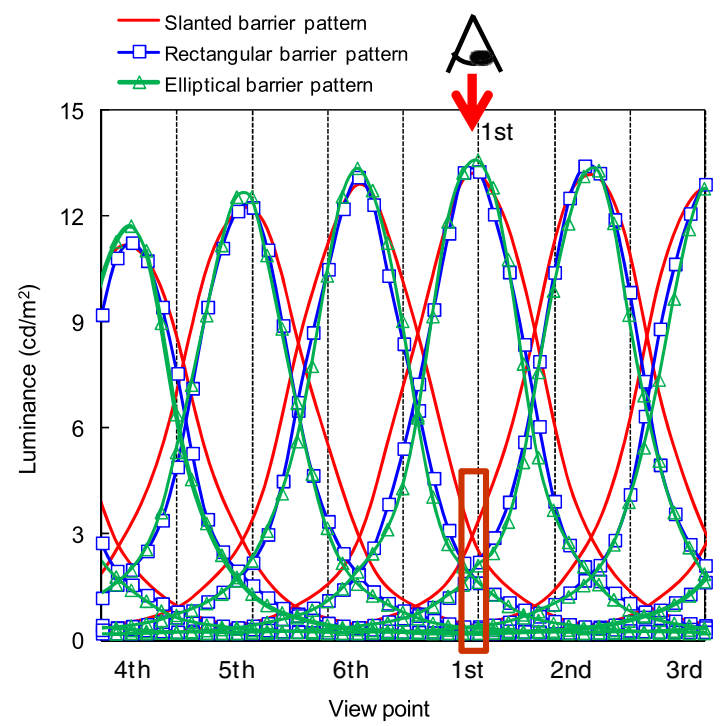

Fig. 8 Measured optical characteristics of the slanted barrier of the width $98 \mu \mathrm{m}$, the rectangular barrier that is $86 \times 262 \mu \mathrm{m}$ and the elliptical barrier that is $98 \times 294 \mu \mathrm{m}$.

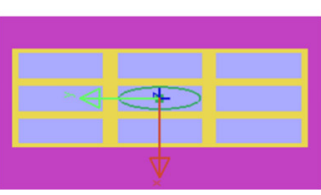

(a)

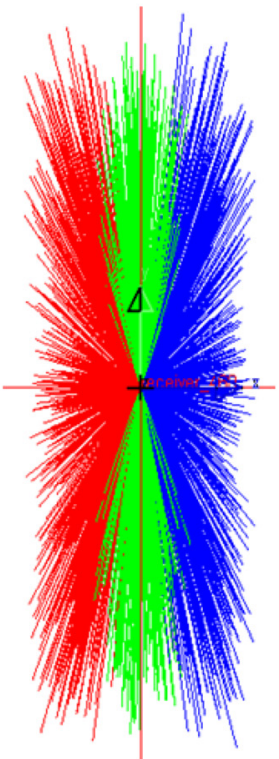

(c)

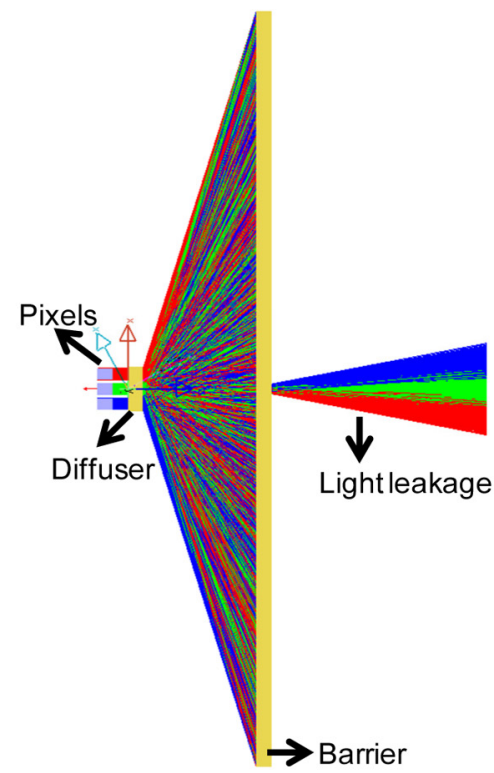

(b)

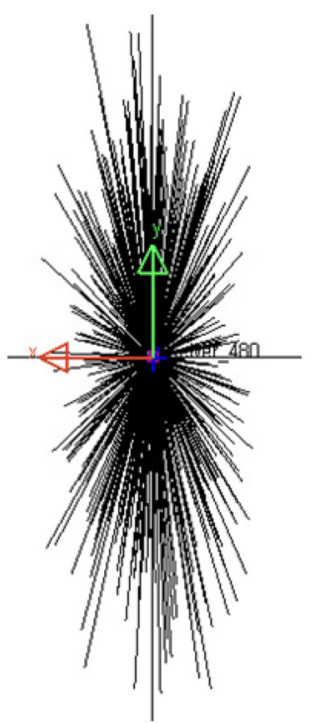

(d)
Fig. 9 Schematic of the simulation. (a) Arrangement of pixels, (b) structure of the simulation (side view), (c) distribution of leakage component at a viewer's position, and (d) distribution of luminance component at a viewer's position. 


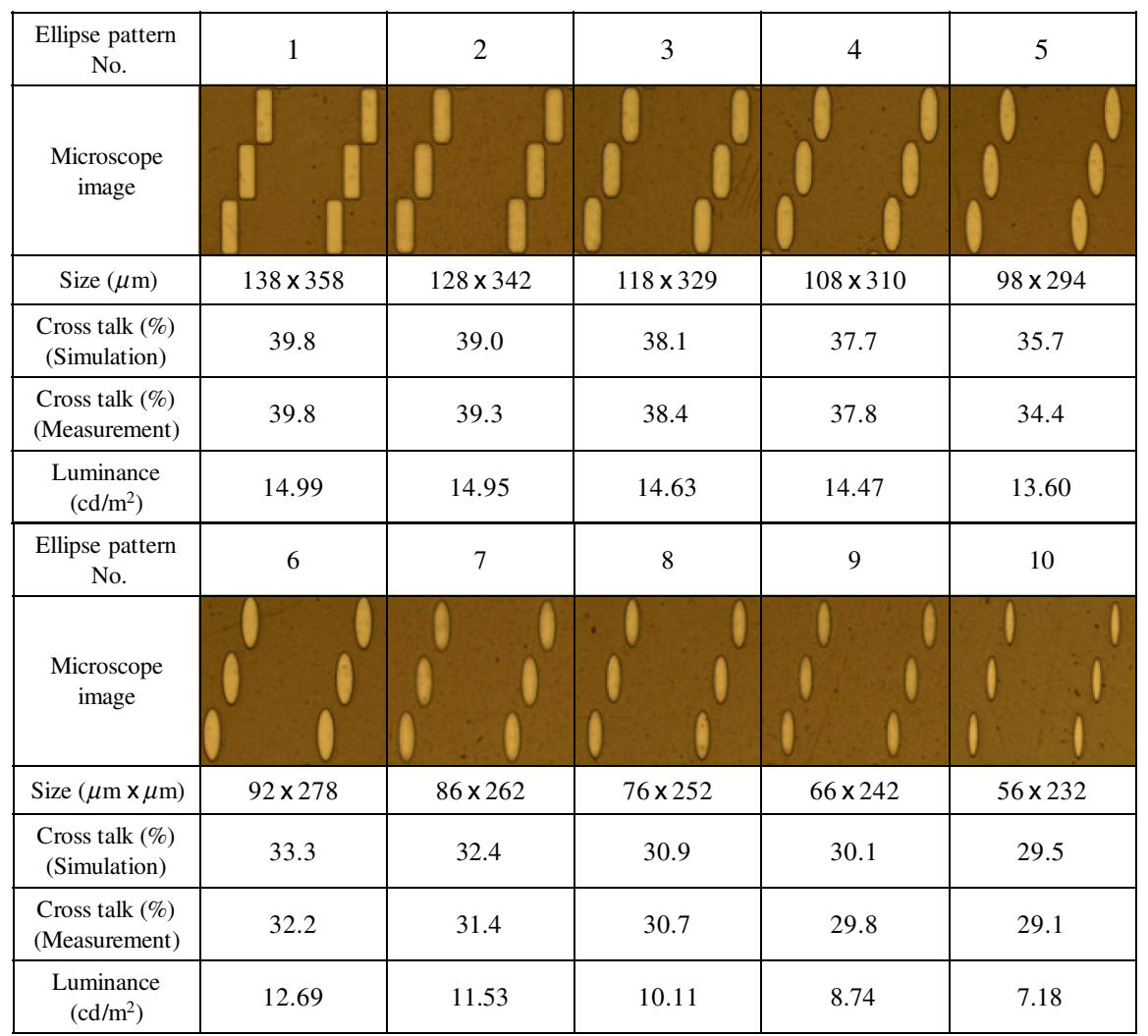

Fig. 10 Microscopic image of our elliptical barrier patterns that varied depending on the lengths of major and minor axes.

horizontal and vertical directions will decrease if the horizontal and vertical lengths of the barrier pattern decrease. The luminance, however, will decrease. Thus, it is important to find the optimum lengths of the barrier pattern. The rectangular pattern is not appropriate to reduce the light leakage from diagonally adjacent pixels. In order to consider cross talk and luminance simultaneously, we propose a new parallax barrier pattern with an elliptical shape. We investigate

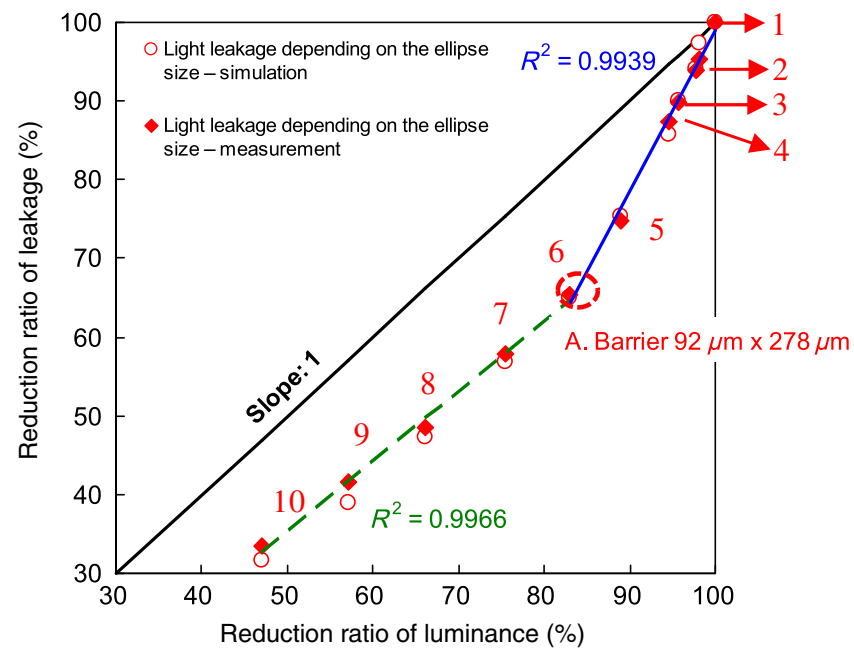

Fig. 11 Relationship between the reduction of light leakage and that of luminance for the various sizes of the elliptical barrier pattern (the numbers in this figure represent the elliptical pattern numbers in Fig. 10). the cross talk carefully taking various lengths of major and minor axes into account.

\section{Results and Discussion}

To verify the effectiveness of the elliptical barrier pattern, we used the six-view autostereoscopic display with various elliptical pattern sizes for our experiments. In addition, we compared the performance of the elliptical barrier pattern with those of the slanted and rectangular barriers.

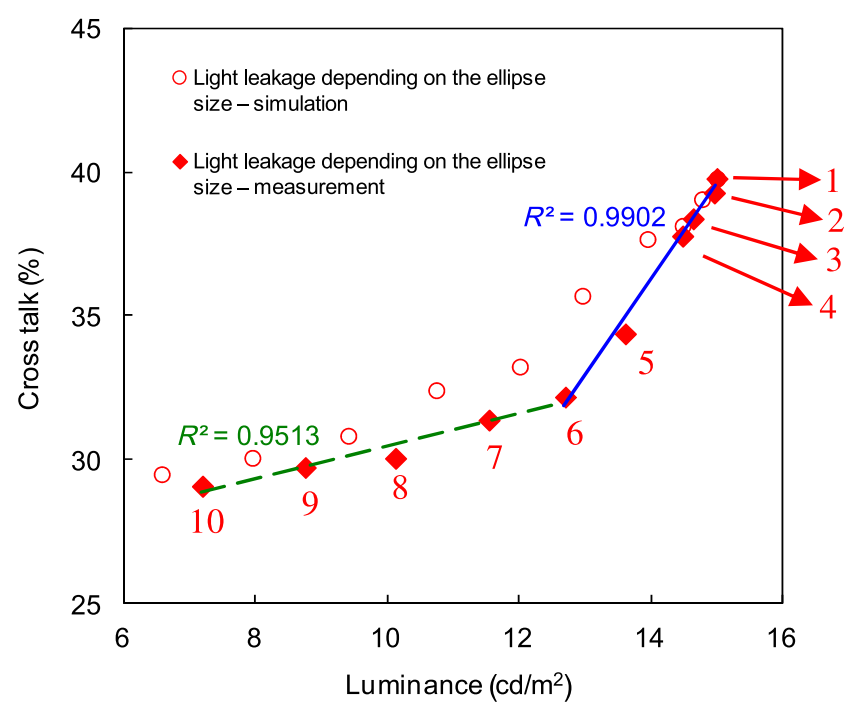

Fig. 12 Relationship between cross talk and luminance (the numbers represent the ellipse pattern numbers in Fig. 10). 


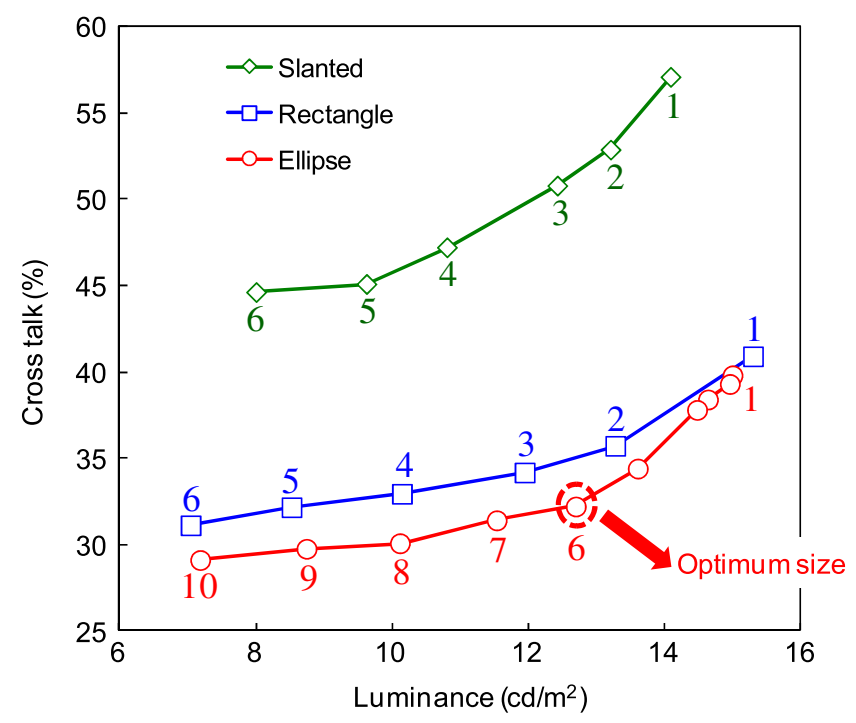

Fig. 13 Overall results of cross talk depending on the luminance at various patterns and sizes.

Figure 5 shows the microscopic images and calculated cross talk of slanted parallax barrier patterns with widths ranging from 53 to $108 \mu \mathrm{m}$. Figure 6 shows the microscopic images and cross talk of the rectangular parallax barrier patterns ranging from $46 \times 222 \mu \mathrm{m}$ to $98 \times 294 \mu \mathrm{m}$, respectively. We can calculate the cross talk described by ${ }^{13}$

Cross $\operatorname{talk}(\%)=\frac{\sum_{i=2}^{6} L_{i} \text { at } 90 \mathrm{deg}}{L_{1} \text { at } 90 \mathrm{deg}} \times 100$.

In Eq. (1), $L_{1}$ and $L_{i}$ represent the luminance of the first view pixels and the luminance of the $i$ 'th view pixels

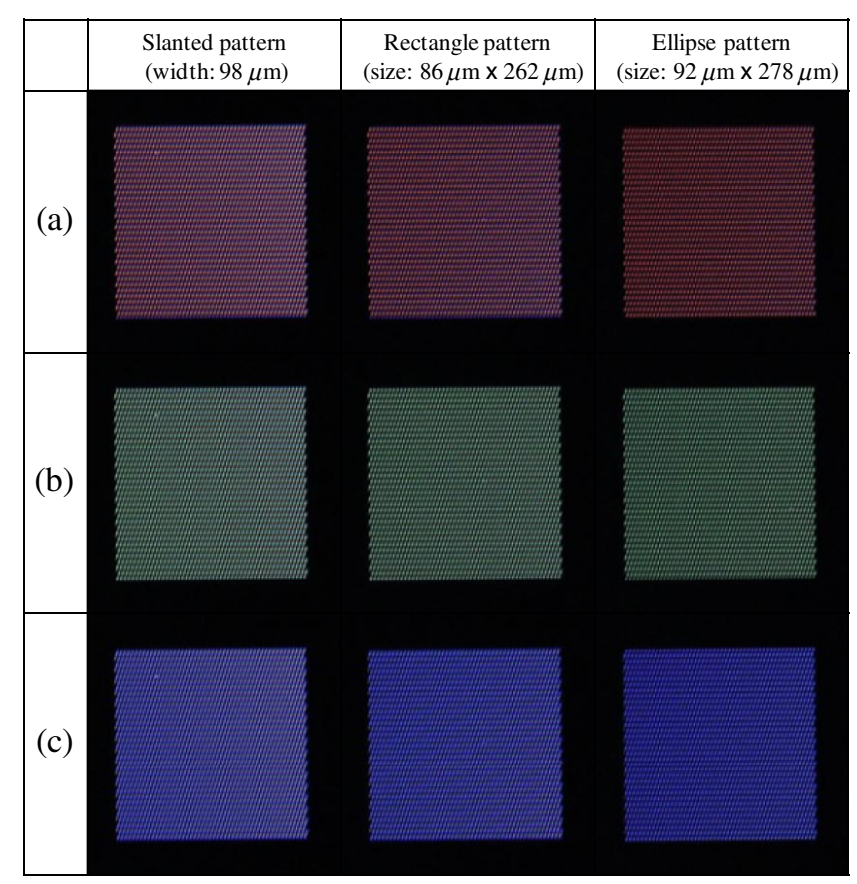

Fig. 14 Actual photos depending on the slanted, rectangular, and elliptical pattern. (a) Red, (b) green, and (c) blue are each displayed at the pixels for the first view, while the pixels for all other views display white. measured at the first view position, respectively. When we measure the cross talk of the first viewpoint, as shown in Fig. 3(b), we fix the color analyzer at the first view's position, and we display white at the pixels for the first view and black at the pixels for the other views. We can measure the first view's luminance. Then, we display white at the pixels for the second view and black at the pixels for the other views. We can measure the second view's cross talk at the first view position. We can measure from the third to the sixth view's cross talk at the first view position in the same way. At this time, color analyzer is still fixed at the first view position. We can obtain precise values because the color analyzer and display panel are both fixed.

Figure 7 shows a summary of how cross talk changes depending on the sizes of both the slanted and rectangular barrier patterns. The results show that a rectangular barrier pattern is superior to a slanted barrier pattern. For example, when we compare the slanted barrier pattern of width of $98 \mu \mathrm{m}$ (No. 2 in Fig. 5) with the rectangular barrier pattern of size $86 \times 262 \mu \mathrm{m}$ (No. 2 in Fig. 6), the cross talk of the rectangular barrier pattern (No. 2 in Fig. 6) has a lower cross talk than that of the slanted barrier pattern (No. 2 in Fig. 5) in spite of the fact that the luminance of the two is similar. This means that the rectangular barrier pattern has a better cross talk performance than the slanted barrier pattern.

Figure 8 shows the measured characteristics of the slanted barrier with the width of $98 \mu \mathrm{m}$ and the rectangular barrier with the area of $86 \times 262 \mu \mathrm{m}$. We display white at the pixels for the first view and black at the pixels for the other views. Then, we move the color analyzer from the fourth view position to the third view position. We can measure the optical characteristics of the first view as shown in Fig. 8. We can measure from the third to the sixth view's optical characteristics in the same way. As shown in Fig. 8, the measured luminance of the first view had a peak value, and the perceived luminance values of the two different displays are similar at the sweet spot. As denoted by solid rectangle in Fig. 8, the cross talk of the rectangular barrier is smaller than that of the slanted barrier pattern.

However, the light leakage from diagonal directions as shown in Fig. 2 was not yet considered. Thus, we propose a new parallax barrier pattern with an elliptical shape to reduce cross talk more effectively by blocking leakage. We investigate cross talk carefully taking various lengths of major and minor axes into account. Here, the major axis means the larger of two axes, which corresponds to the largest distance between antipodal on the ellipse, whereas the minor axes means the one with the smallest distance across the ellipse. When the lengths of both the major and minor axes are short, the cross talk caused by light leakage is improved while the luminance of the panel is not. It always suffers from the trade-off between brightness and cross talk. Therefore, finding the optimum lengths of the ellipse pattern is important. In order to find it, we investigated the relationship between the cross talk and the length of the elliptical pattern with an optical simulator, light tools. Figure 9 shows a schematic of an optical simulation. We arranged 9 pixels as shown in Fig. 9(a). The pixels have the same size as those of the LCD panel used in the previous experiment. Figure 9(b) shows the structure of the optical simulation seen from the side view. The diffuser makes the light spread as a Lambertian distribution. Figures 9(c) 


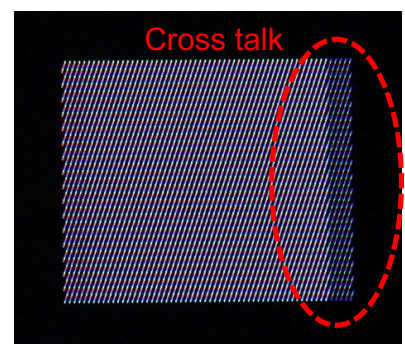

(a)

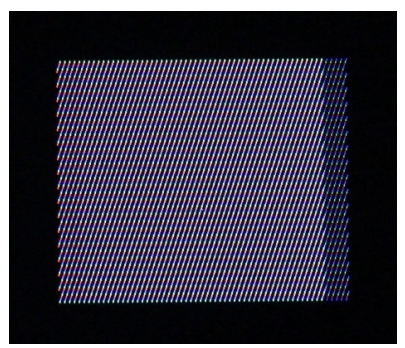

(b)

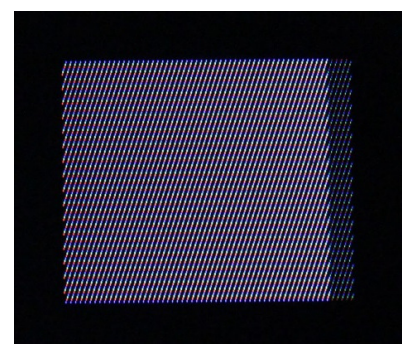

(c)

Fig. 15 Actual photos of the 3-D rectangular image with a horizontal disparity of 30 pixels when we used (a) slanted, (b) rectangular, and (c) elliptical patterns.

and 9(d) show the distributed light rays of leakage component and luminance component from the optical simulation. We simulated the various sizes of elliptical barrier patterns as shown in Fig. 10, and we calculated the cross talk using the ratio of the number of light rays for light leakage component to luminance component.

We fabricated the elliptical barrier pattern. Figure 10 shows the microscopic image of elliptical barriers with the various lengths of major and minor axes. When the length of the major and minor axes was larger than the pixel size $(98 \times 294 \mu \mathrm{m})$, we blocked the area of the ellipse that was larger than the pixel size to prevent it invading the area of its adjacent pixel. We need to find the relationship between the cross talk and various lengths of the major and minor axes of the elliptical barriers. First, we analyzed the relationship between the reduction of light leakage and as reduction of luminance depending on the size of the ellipses as shown in Fig. 11. The black solid line is introduced as a reference with a slope of 1 . Thus, if the slope of data is higher than 1 , the reduction ratio of leakage is larger than that of luminance, which is more efficient. The red circles and solid diamonds show the simulation and measurement results for each size of ellipse, respectively. The blue solid and green dashed lines are the fitting lines with different slopes. As shown in Fig. 11, we find that the blue solid line is more efficient than the green dashed line because the slope of the blue line is higher than 1 while the green line is almost 1. From the results of Fig. 11, we can deduce that the optimum size of the ellipse is $92 \times 278 \mu \mathrm{m}$. Second, we analyzed the relationship between cross talk and luminance in the same way as shown in Fig. 12. The reduction of cross talk for the blue line is steeper than that of the green line, which means that the optimum size was also the same as was determined by Fig. 11. From these results, we can deduce that the optimum size of the ellipse is $92 \times 278 \mu \mathrm{m}$. Figure 13 shows the overall result of how the cross talk depends on the luminance as well as the pattern and size. As shown in Fig. 13, the elliptical pattern shows superior cross talk characteristics when compared to the slanted pattern, and the cross talk is further reduced than the rectangular pattern. The cross talk of the elliptical barrier with the optimum size of $92 \times 278 \mu \mathrm{m}$ was about $32 \%$, whereas the slanted and rectangular one are about $57 \%$ and $36 \%$ cross talk, respectively, at the same luminance.

Table 1 Simulation conditions depending on pixel sizes.

\begin{tabular}{|c|c|c|c|c|c|c|c|}
\hline \multicolumn{3}{|r|}{ Condition } & 1 & 2 & 3 & 4 & 5 \\
\hline \multicolumn{3}{|r|}{ Pixel size } & $88 \mu \mathrm{m} \times 264 \mu \mathrm{m}$ & $93 \mu \mathrm{m} \times 279 \mu \mathrm{m}$ & $98 \mu \mathrm{m} \times 294 \mu \mathrm{m}$ & $103 \mu \mathrm{m} \times 309 \mu \mathrm{m}$ & $108 \mu \mathrm{m} \times 324 \mu \mathrm{m}$ \\
\hline 1 & $100 \%$ & $\begin{array}{l}\text { Ratio of the area of } \\
\text { the ellipse to pixel }\end{array}$ & $88 \mu \mathrm{m} \times 264 \mu \mathrm{m}$ & $93 \mu \mathrm{m} \times 279 \mu \mathrm{m}$ & $98 \mu \mathrm{m} \times 294 \mu \mathrm{m}$ & $103 \mu \mathrm{m} \times 309 \mu \mathrm{m}$ & $108 \mu \mathrm{m} \times 324 \mu \mathrm{m}$ \\
\hline \multirow[t]{2}{*}{2} & $90 \%$ & & $98 \mu \mathrm{m} \times 294 \mu \mathrm{m}$ & $103 \mu \mathrm{m} \times 310 \mu \mathrm{m}$ & $109 \mu \mathrm{m} \times 328 \mu \mathrm{m}$ & $115 \mu \mathrm{m} \times 345 \mu \mathrm{m}$ & $120 \mu \mathrm{m} \times 360 \mu \mathrm{m}$ \\
\hline & $80 \%$ & & $89 \mu \mathrm{m} \times 297 \mu \mathrm{m}$ & $94 \mu \mathrm{m} \times 293 \mu \mathrm{m}$ & $99 \mu \mathrm{m} \times 298 \mu \mathrm{m}$ & $104 \mu \mathrm{m} \times 312 \mu \mathrm{m}$ & $109 \mu \mathrm{m} \times 327 \mu \mathrm{m}$ \\
\hline 4 & $70 \%$ & & $83 \mu \mathrm{m} \times 249 \mu \mathrm{m}$ & $88 \mu \mathrm{m} \times 263 \mu \mathrm{m}$ & $92 \mu \mathrm{m} \times 278 \mu \mathrm{m}$ & $97 \mu \mathrm{m} \times 291 \mu \mathrm{m}$ & $102 \mu \mathrm{m} \times 306 \mu \mathrm{m}$ \\
\hline 5 & $60 \%$ & & $77 \mu \mathrm{m} \times 231 \mu \mathrm{m}$ & $81 \mu \mathrm{m} \times 245 \mu \mathrm{m}$ & $86 \mu \mathrm{m} \times 258 \mu \mathrm{m}$ & $90 \mu \mathrm{m} \times 270 \mu \mathrm{m}$ & $94 \mu \mathrm{m} \times 282 \mu \mathrm{m}$ \\
\hline 6 & $50 \%$ & & $70 \mu \mathrm{m} \times 212 \mu \mathrm{m}$ & $74 \mu \mathrm{m} \times 222 \mu \mathrm{m}$ & $78 \mu \mathrm{m} \times 235 \mu \mathrm{m}$ & $82 \mu \mathrm{m} \times 246 \mu \mathrm{m}$ & $86 \mu \mathrm{m} \times 258 \mu \mathrm{m}$ \\
\hline 7 & $40 \%$ & & $63 \mu \mathrm{m} \times 190 \mu \mathrm{m}$ & $66 \mu \mathrm{m} \times 200 \mu \mathrm{m}$ & $70 \mu \mathrm{m} \times 210 \mu \mathrm{m}$ & $74 \mu \mathrm{m} \times 220 \mu \mathrm{m}$ & $77 \mu \mathrm{m} \times 231 \mu \mathrm{m}$ \\
\hline 8 & $30 \%$ & & $55 \mu \mathrm{m} \times 164 \mu \mathrm{m}$ & $57 \mu \mathrm{m} \times 173 \mu \mathrm{m}$ & $61 \mu \mathrm{m} \times 182 \mu \mathrm{m}$ & $64 \mu \mathrm{m} \times 192 \mu \mathrm{m}$ & $67 \mu \mathrm{m} \times 201 \mu \mathrm{m}$ \\
\hline \multicolumn{3}{|c|}{ Gap between the panel and barrier } & $0.678 \mathrm{~mm}$ & $0.716 \mathrm{~mm}$ & $0.75 \mathrm{~mm}$ & $0.792 \mathrm{~mm}$ & $0.829 \mathrm{~mm}$ \\
\hline
\end{tabular}

Viewing distance

$50 \mathrm{~cm}$ 


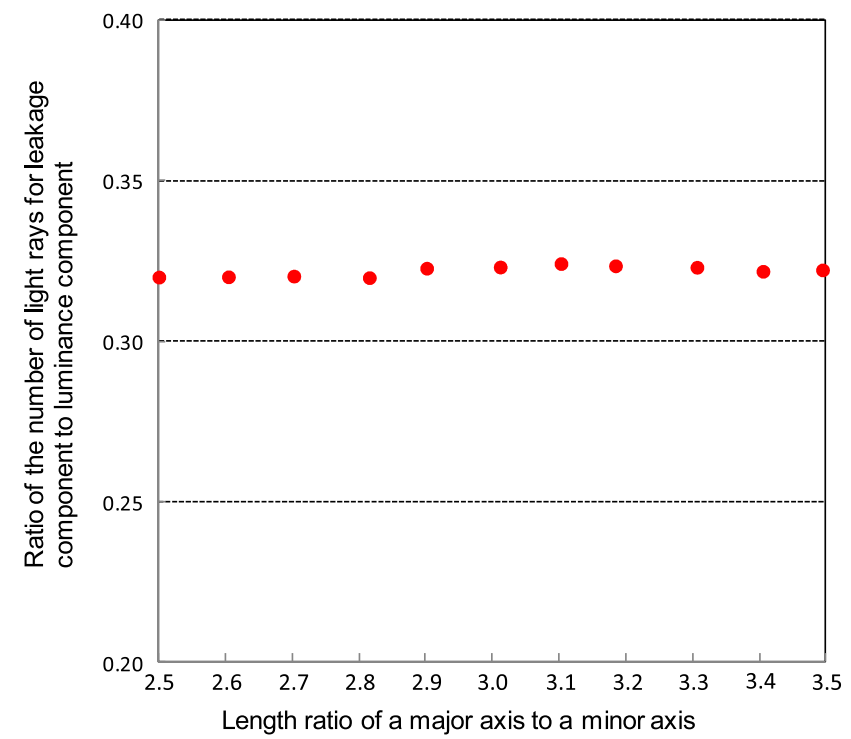

Fig. 16 Ratio of the leakage component to luminance component according to the length ratio of a major axis to a minor axis of the ellipse with aperture area $18,800 \mu \mathrm{m}^{2} /$ pixel.

As a result, we can obtain the best performance with an elliptical barrier pattern out of all the barriers at the same luminance. Furthermore, we can also deduce that having a barrier size smaller than $92 \times 278 \mu \mathrm{m}$ is inefficient in terms of cross talk reduction due to the excessive luminance reduction.

We took actual photos depending on the shapes of barriers as shown in Fig. 14. Figure 14(a) represents the actual photos taken when we displayed a red box at the pixels for the first view and a white box at the pixels for the other views. Figures 14(b) and 14(c) show the photos for green and blue, respectively. We used slanted, rectangular, and elliptical patterns with widths $98 \mu \mathrm{m}$, size of $86 \times 262 \mu \mathrm{m}$, and $92 \times 272 \mu \mathrm{m}$, respectively. Measured luminance was almost the same. However, we can see more saturated red, green, and blue colors from the display with the elliptical pattern

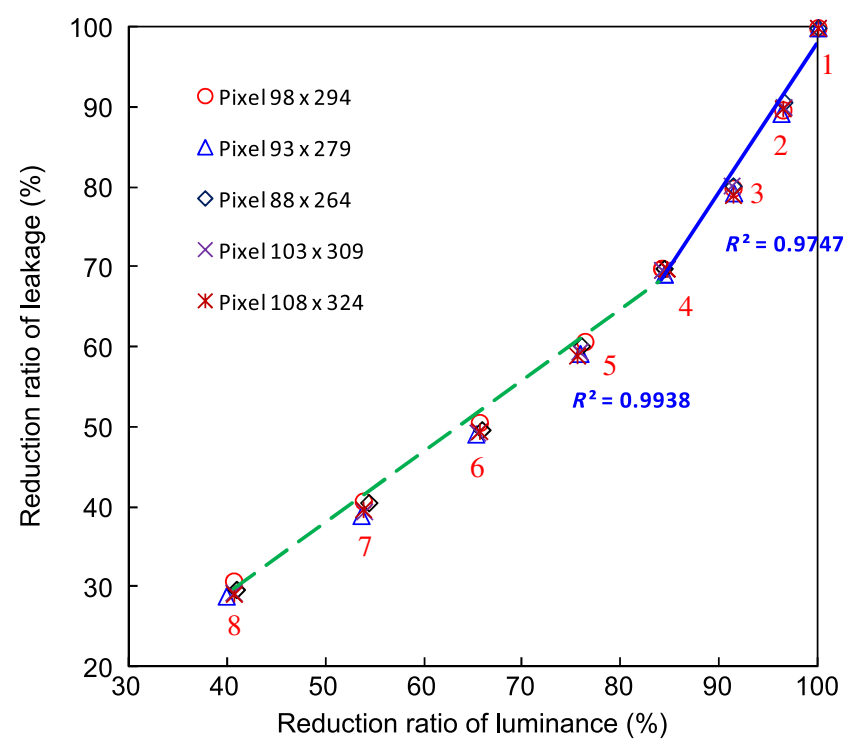

Fig. 17 Relationship between the reduction of light leakage and that of luminance depending on the pixel size (the numbers in this figure represent the elliptical pattern numbers in Table 1).

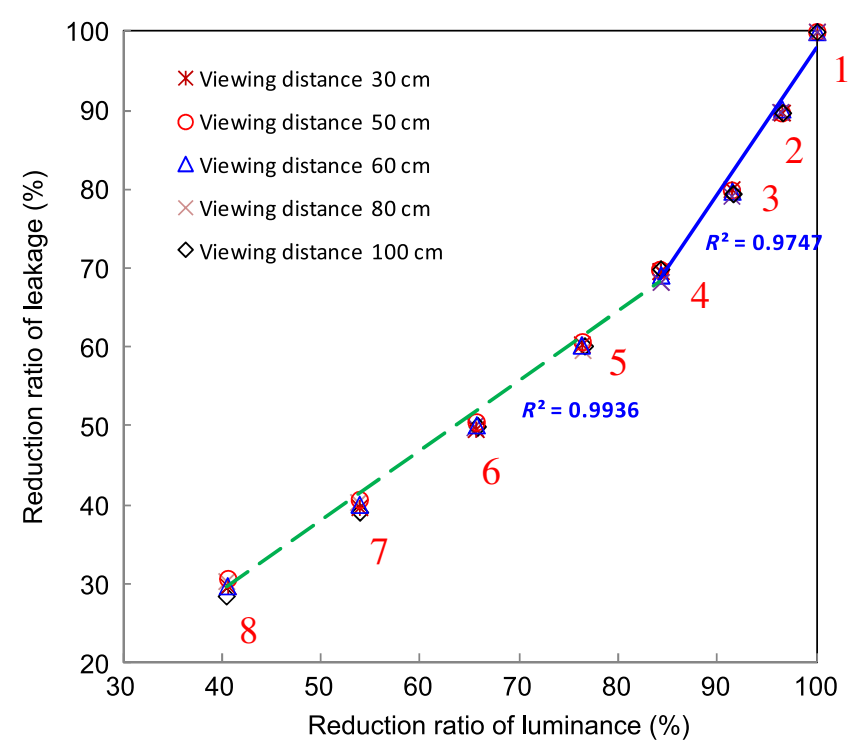

Fig. 18 Relationship between the reduction of light leakage and that of luminance depending on the viewing distance (the numbers in this figure represent the elliptical pattern numbers in Table 1).

than the other ones due to reduced cross talk. Thus, we can prove that actual cross talk is decreased. Figure 15 shows the photos taken from the 3-D rectangular image when the horizontal disparity was 30 pixels. We took photos at the location of the right eye. Thus, the cross talk appeared at the right side of the rectangular image because the viewer cannot help perceiving the left image. As shown in Fig. 15(c), we can see that the cross talk region of Fig 14(c) is darker than those of Figs. 14(a) and 14(b). In addition, we simulated to find an optimum size depending on the pixel size and viewing distance. First, as shown in Table 1, we simulated five conditions of different pixel sizes. In the simulation, we varied the sizes of the ellipse according to the ratios of the area of the ellipse to pixel area. For example, the area of the ellipse with the $92 \times 278 \mu \mathrm{m}$ is $70 \%$ of the area of the pixel with the $98 \times 294 \mu \mathrm{m}$. The gap between the barrier and the panel was adjusted according to the pixel area so that the viewing distance may be fixed to $50 \mathrm{~cm}$. We can actually observe that the ray ratio of light leakage is almost constant regardless of the length ratio of a major axis and minor axis as shown in Fig. 16. Thus, we fixed the length ratio of the major and minor axes to $3: 1$, the aspect ratio of the LCD. Figure 17 shows the simulation results. The reduction ratio of leakage for $70 \%$ to $100 \%$ of the pixel area is larger than that of luminance, whereas the reduction ratio of leakage for the area smaller than $70 \%$ of the pixel area is the same as that of luminance. We can deduce that the most efficient area of the ellipse is $70 \%$ of the pixel area to reduce the cross talk.

Second, we varied the viewing distance to find an optimum condition. The gap between the barrier and the panel was adjusted viewing distances to $30,50,60,80$, and $100 \mathrm{~cm}$ when the pixel size is $98 \times 294 \mu \mathrm{m}$. As shown in Fig. 18, we can find that the most efficient area of the ellipse is $70 \%$ of the pixel area to reduce the cross talk regardless of viewing distance. In summary, the optimum area of the barrier is $70 \%$ of the pixel area regardless of pixel sizes and viewing distances to reduce the cross talk. 


\section{Conclusion}

In this paper, we propose a new parallax barrier pattern with an elliptical shape that reduces the cross talk caused by light leakage from all the adjacent subpixels. Because of the tradeoff between the luminance and cross talk in a parallax barrier-type 3-D display, we analyzed the relationship between the reduction of cross talk and that of luminance depending on the size of the elliptical pattern. Through this relationship, we optimized the size of the proposed barrier pattern. In addition, we verified that the cross talk of the proposed barrier pattern was superior to both the conventional slanted and rectangular barrier patterns at the same luminance condition. We think that our proposed barrier pattern can be applied to autostereoscopic displays as a design factor for reducing cross talk. We expect that an autostereoscopic display with our proposed barrier will provide much better cross talk reduction for viewers.

\section{Acknowledgments}

This work was supported by the National Research Foundation of Korea Grant funded by the Korean government (KRF 2013-022887).

\section{References}

1. J.-Y. Luo et al., "Two-parallax-barriers-based autostereoscopic liquidcrystal display without crosstalk," J. SID 19(10), 675-678 (2011).

2. W.-X. Zhao et al., "Pixel arrangement of autostereoscopic liquid crystal displays based on parallax barriers," Mol. Cryst. Liq. Cryst. 507(1), 67-72 (2009).

3. N. A. Dodgson, "Analysis of the viewing zone of multi-view autostereoscopic displays," Proc. SPIE 4660, 254-265 (2002).

4. R.-P. M. Berretty, F. J. Peters, and G. T. G. Volleberg, "Real time rendering for multiview autostereoscopic displays," Proc. SPIE 6055, 60550N (2006).

5. X.-F. Li et al., "Image processing to eliminate crosstalk between neighboring view images in three-dimensional lenticular display," J. Disp. Technol. 7(8), 443-447 (2011).

6. C.-H. Chen et al., "Liquid crystal panel for high efficiency barrier type autostereoscopic three-dimensional displays," Appl. Opt. 48(18), 3446-3454 (2009).

7. J. Kim et al., "User-friendly minimization technology of threedimensional crosstalk in three dimensional liquid crystal display televisions with active shutter glasses," Opt. Eng. 51(10), 107401 (2012).

8. L. Wang et al., "Crosstalk evaluation in stereoscopic displays," J. Disp. Technol. 7(4), 208-214 (2011).

9. F. Kooi and A. Toet, "Visual comfort of binocular and 3D displays," Displays 25(2-3), 99-108 (2004).

10. F. Kooi and A. Toet, "Visual comfort of binocular and 3D displays," Displays 25(2-3), 99-108 (2004).

11. R. Fukushima et al., "Effect of light ray overlap between neighboring parallax images in autostereoscopic 3D displays," Proc. SPIE 7237, 72370W (2009).
12. Y. Takaki, "Multi-view 3-D display employing a flat-panel display with slanted pixel arrangement," J. SID 18(7), 476-482 (2010).

13. S. K. Kim, S. K. Yoon, and K. H. Yoon, "Crosstalk minimization in autostereoscopic multiview 3D display by eye tracking and fusion(overlapping) of viewing zones," Proc. SPIE 8384, 838410 (2012).

14. K. Mashitan, H. Takahash, and T. Aida, "Multi-view glass-less 3-D display by parallax barrier of step structure," Mem. Fac. Eng. Osaka City Univ. 48, 1-8 (2007).

15. E. Lueder, $3 D$ Displays, p. 95 , John Wiley \& Sons Inc, New York (2012).

Yongsik Jung received his BS degree in electric engineering at Korea University, Republic of Korea, in 2004. He is a senior engineer at Samsung Display, and is currently working toward his MS degree with the Department of Information Display at Kyung Hee University. His research interests include driving methods and circuits for OLED displays and LCDs.

Jong-Man Kim received his BS and MS degrees in physics and information display at Kyung Hee University, Korea, in 2010 and 2012, respectively. He is currently working toward his $\mathrm{PhD}$ in the Department of Information Display at Kyung Hee University. His research interests include driving methods and circuits for LCD and e-paper displays and driving technology for color motion performance of LCDs.

Jongbin Kim received his BS and MS degrees in physics and information display at Kyung Hee University, Korea, in 2010 and 2012, respectively. He is currently working toward his $\mathrm{PhD}$ in the Department of Information Display at Kyung Hee University. His research interests include driving methods and circuits for LCD and OLED displays and driving technology for color motion performance of LCDs.

Hyunsik Sung received his BS degree in the Department of Information Display from Kyung Hee University, Seoul, Korea, in 2012. In 2012, he started working on his MS degree. His current research interests focus on the display optical application and the human factor related on three-dimensional (3-D) display.

Sung-Wook Min received the BS and MS degrees in electrical engineering from Seoul National University, Korea, in 1995 and 1997, respectively. In August 2004, he received the PhD degree from his alma mater. Currently, he is a faculty member in the Department of Information Display, Kyung Hee University, which he joined in 2007. He is interested in 3-D imaging and the advanced display system, especially based on the integral imaging technique.

Seung-Woo Lee received his MS and PhD degrees from KAIST in electrical engineering in 1995 and 2000, respectively. He joined Samsung in 2000, where his work has focused on the development of key driving technologies for active-matrix liquid-crystal displays. He is currently an associate professor in the Department of Information Display at Kyung Hee University. He has been active with SID as a senior member. He became an IEEE senior member in 2010. 